\title{
Clustering Artificial Atoms Induced by High-Frequency Electromagnetic Radiation in Graphene Monolayers of Multiwalled Carbon Nanotubes
}

\author{
H. V. Grushevskaya, ${ }^{*}$ A. I. Timoshchenko, ${ }^{\dagger}$ E. A. Avdanina, and I. V. Lipnevich \\ Physics Department, Belarusian State University, \\ 4 Nezalezhnasti Ave., 220030 Minsk, BELARUS
}

(Received 26 September, 2020)

\begin{abstract}
A graphene-charge carrier confinement induced by high-frequency photons and a subsequent clustering of artificial atoms in graphene plane have been studied using electrophysical and Raman-spectroscopy methods. To fabricate the graphene n-p-n junctions, commensurable superlattice structures consisting of multi-walled carbon nanotubes (MWCNTs) have been formed utilizing a Langmuir-Blodgett technique. It has been shown that the $\mathrm{p}-\mathrm{n}$ graphene junctions are sensitive to graphene lattice-deformation defects only. The levels of graphene defect do not host impurity electrons. One offers a mechanism of graphene monolayer self-repairing after a radiation damage. This mechanism is based on an existence of topologically protected Compton scatterers in graphene plane.
\end{abstract}

PACS numbers: $05.60 . \mathrm{Gg}, 72.80 . \mathrm{Vp}, 73.22$

Keywords: radiation-induced graphene p-n junction, Majorana-like fermion charge carriers carbon nanotube

DOI: https://doi.org/10.33581/1561-4085-2020-23-3-342-356

\section{Introduction}

Graphene, carbon nanotubes (CNT), and graphene-like materials can exhibit radiation resistance and are promising structural materials for nuclear applications, for example, new generation of photodetectors [1] and as a replacement for plastic beta-scintillators [2] due to the following features. Band structure of graphene materials is gapless, their bands are touched, and chiral charge carriers are massless. Lindhard atom screening [3] happens in graphene and ultrathin crystal so that noninteracting with electrons incoming particles are scattered by a crystalplane atom potential. Subsequently a fast surface diffusion of knocked-out $\mathrm{C}$ atoms proceeds very fast in the vacancies places (vacancy annealing) [4]. The atoms, which are knocked out of the graphene plane or ultrathin crystal under action of high-energy $(1 \mathrm{MeV}-1 \mathrm{GeV})$ ions [5-7],

\footnotetext{
${ }^{*}$ E-mail: grushevskaja@bsu.by

${ }^{\dagger}$ E-mail: a_timoshchenko@mail.ru
}

electrons (see [6, 8-10] and references therein), protons and positrons (see $[6,11]$ and references therein), cannot escape from the graphene plane to enter the deep bulk region. Emerging in beta-rays-,ions-irradiated graphene the radiation vacancies form clusters. $[8,12,13]$ The vacancies are neutral, but they host a positively charge by applying electromagnetic pulses [13].

As well, radiation resistivity of MWCNTs is revealed as a possibility for carbon atoms to be displaced by gamma-rays in the vicinity of graphene plane only that a radiation structural rearrangement is restricted to chemical cross link of the nearest carbon nanotubes by formation of chemical bonds between neighbor $\mathrm{C}$ atoms from graphene layers or between $\mathrm{C}$ atoms on the interface CNT-environment [7, 12]. However, the relation between the ability of vacancies to cluster formation and the ability of multiwalled CNTs (MWCNTs) to self repair is not clearly understood.

Chiral massless charge carriers in graphene form electron-hole pairs. An electron-hole density in the Dirac point $K\left(K^{\prime}\right)$ of graphene Brillouin 
zone is extremely high due to the fact that the energy of electron-hole pair is zero. The value of hole/electron density is high enough to provide trapping of bombarding negatively/positively electrically charged particle. The last will also be impeded to channeling of $1 \mathrm{MeV}-1 \mathrm{GeV}$ electrons and consequently to a generation of $X$ - and $\gamma$ rays, stimulated by the curvature of CNTs or similar periodically-bent crystalline structures. The presented mechanism of trapping is also in agreement with the absence of experimental facts about such type of generation [14] theoretically predicted in $[15,16]$. Because of the gapless structure, the interaction between graphene and electro-magnetic radiation presents itself an elastic scattering of gamma-quanta on free charge carriers. Notwithstanding large Compton effect, an ultrafast hot carrier recombination on a sub-picosecond timescale attenuates sharply the response of modern graphene broadband photodetectors [17].

So, the most fundamental radiation damage studies, such as many-body surface-defect evolution processes, are lacking.

In the paper we study the influence of weak flows of gamma- and beta-ray particles from radiation source of ${ }^{137} \mathrm{Cs}$ on electrophysical properties of ultra-thin Langmuir-Blodgett (LB) films which have been fabricated from MWCNTs decorated by organometallic compound. We demonstrate that the electrophysical properties of LB-films are recovered after irradiation. Moreover, shielding effect of the high-conductivity MWCNT LB-bundles is sharply enhanced. To discover a mechanism of graphene radiation resistance we will also utilize laser pulses to produce the n-p-n junctions of $\mathrm{p}$-doped and n-doped patches in graphene-like materials of different types. The junctions induced by the hight-frequency electromagnetic radiation will be investigated by Raman spectroscopy methods.

\section{Methods}

\subsection{Sample fabrication}

Carboxylated and stearic-acid-functionated MWCNTs under 2.5-5 $\mathrm{nm}$ in diameter have been decorated by nanocyclic complexes (Fe(II)DTP) of Ce and/or high-spin octahedral Fe with ligands being conducting oligomer of dithionylpyrrole series in the following way. As a preliminary, an alkyl hydrocarbon chain $\mathrm{C}_{16} \mathrm{H}_{33}$ was linked chemically to the oligomer. Chemical formula of the oligomer has the following form: 3-hexadecyl2,5-di(thiophen-2-yl)-1H-pyrrole (H-DTP). A 5 -monolayer graphene-like film of the nanocyclic organometallic complexes is fabricated by means of LB-nanotechnique. Then, inverse micelles of stearic acid with MWCNTs inside are obtained by mixing stearic acid and MWCNTs in hexane by the ultrasound treatment, and 2-monolayer MWCNT films fabricated from these micellar MWCNTs by LB-nanotechnique are deposited on the nanocyclic-complex LB-cover. The MWCNT LB-bundles nondecorated/decorated by organometallic complexes or the LB-films of nanocyclic organometallic complexes were deposited on the interdigital structure of aluminium electrodes, on the surface of which a layer of nanoporous anodic alumina (AOA) were previously formed as a insulator coating.

\subsection{Supports modifications}

Hydrophilization of Si surface was performed by its treating with mixture of sulphuric acid $\left(\mathrm{H}_{2} \mathrm{SO}_{4}\right)$ and hydrogen peroxide $\left(\mathrm{H}_{2} \mathrm{O}_{2}\right)$ for 30 min. It ordinary is used to remove organic residues from a substrate. After that, the Si plate was washed out in deionized water followed by the ultrasound treatment for $10 \mathrm{~min}$. Meanwhile, additionally to the organic substrates were removed from the Si surface, a hydrophilic film of $\mathrm{SiO}_{2}$ is formed with very flat surface [18] The surface of nanoporous anodic alumina is hydrophilic. For its hydrophobization the solution of H-DTP in hexane was dropped on the surface. 


\subsection{Exposure to radiation}

A low-intensive source of ionizing radiation (IRS) ${ }^{137} \mathrm{Cs}$ (CsJ) with activity of radioactive decay $A$ about $6.22 \times 10^{4} \mathrm{~Bq}$ at the time of investigation. Beta-decay of unstable nuclei ${ }^{137} \mathrm{Cs}$ is accompanied by emission of beta-particles with the energies $0.512,0.892$, and $1.176 \mathrm{MeV}$ and by production of Ba nuclei. $94.7 \%$ of Ba nuclei being in metastable state emit gamma-quanta with mean energy $662 \mathrm{keV} \mathrm{[19].} \mathrm{The} \mathrm{radiation} \mathrm{of} \mathrm{low-}$ intensive beta-particles beam has been attenuated by thin-film aluminium screen. The irradiation dose was chosen to be $10^{-9} \mathrm{~Gy} /$ hour. The IRS has the form of a drop with average diameter about $d=1.5 \mathrm{~mm}$. A sample with diameter $D_{s}=4 \mathrm{~mm}$ is exposed through collimator being of the order of $5 \mathrm{~mm}$ in diameter and $L=50 \mathrm{~mm}$ long. A scheme of irradiation is shown in fig. 1. At ratio $d / L=0.03$, IRS can be considered as a point source.

A scintillation detector has been constructed based on $\mathrm{NaJ}(\mathrm{Te})$ crystal having diameter of $25 \mathrm{~mm}$ and hight of $40 \mathrm{~mm}$. A fluence rate of nonscattered gamma-radiation within the irradiated sample can be estimated as

$$
\phi_{0}=\frac{k A}{4 L^{2}} \frac{\pi D_{s}^{2}}{4 L^{2}}
$$

where $k$ is a percentage of emitted photons per one decay (so called quantum yield), $\frac{\pi D_{s}^{2}}{4 L^{2}}$ is a solid angle under which the irradiated sample is viewed from the point of IRS. For IRS ${ }^{137} \mathrm{Cs}$ $k=0.851$. Therefore $\phi_{0}=8.5 \times 10^{3} \mathrm{~m}^{-2} \mathrm{c}^{-1}$. A number of $\gamma$-quanta coincides with a number of $0.512 \mathrm{MeV}$ electrons emitted by ${ }^{137} \mathrm{Cs}$ atoms in decay process. Since the percentage of $1.174 \mathrm{MeV}$ electrons is $5.3 \%$, the fluence of beta-quanta with the energy $1.174 \mathrm{MeV}$ is less than $485 \mathrm{~m}^{-2} \mathrm{c}^{-1}$. Irradiation of the organometallic LB-films has been performed for 1 hour, MWCNTs were exposed to radiation for 1 or 3 hours. A graphene monolayer deposited on the insulator surface which becomes electric under the action of $\beta$ radiation [32] is doped. To avoid parasitic doping, the graphene materials suspended on nanoporous AOA were irradiated only.

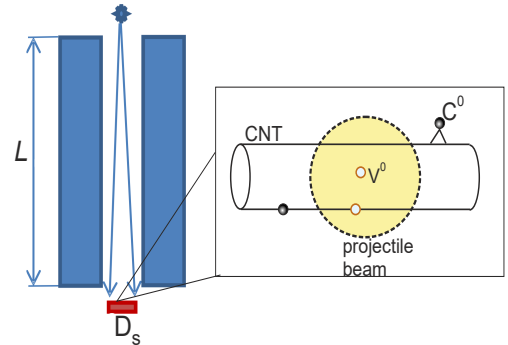

FIG. 1. (color online) A scheme of irradiation of sample (underneath the collimator of length $L$ ) of diameter $D_{s}$. IRS is placed above the collimator. Inset : Sketch of radiation defects presents creation of neutral vacancies $V^{0}$ with knocked-out neutral atoms $\mathrm{C}^{0}$ fixed on nanotube wall (graphene monolayer).

Samples are exposed to laser radiation at light wavelengths 355, 473, and $532 \mathrm{~nm}$ with power in range from 0.0001 to $20 \mathrm{~mW}$ at room temperatures (RT).

\subsection{Impedance measurements}

We use the planar capacitive sensor of interdigital-type on such glass-ceramic support as pyroceramics [20]. $N$ pairs, $N=20$ of aluminium electrodes of the sensors are arranged in an Archimedes-type spiral configuration. Every such pair is an "open type" capacitor. The dielectric coating of the electrodes represents itself nanoporous anodic alumina with a pore diameter of $10 \mathrm{~nm}$. The synthesized LB-nanoheterostructures were suspended on the interdigital electrode structure. To excite harmonic auto-oscillations of electric current (charge-discharge (charging) processes in the capacitors), the sensor was connected as the capacitance $C$ into the relaxation resistance $(R)$-capacitance $(C)$ oscillator (self-excited RCoscillator) [21]. Operating of such RC- oscillator is based on the principle of self-excitation of an amplifier with a positive feedback on quasiresonance frequencies. The capacitance $C$ of the sensor entered in measuring RC-oscillating circuit has been calculated by the formula $C=1 /(2 \pi R f)$, where $R$ is the measuring 
resistance, $f$ is a quasi-resonance frequency. Electrophysical properties of the ultra-thin LBfilms were studied by means of the impedance spectroscopy methods as a variation in dielectric polarization of double electrically charged layer formed at the interface between a surface of the fabricated electrochemical sensor and water. Electrochemical measurements were carried out in deionized water at RT.

\subsection{Raman spectroscopy studies}

Spectral studies in visible range were carried out using a confocal micro-Raman spectrometer Nanofinder HE ("LOTIS-TII", Tokyo, JapanBelarus).

\subsection{Theoretical modeling}

A quasi-relativistic graphene model Hamiltonian reads [22-25]

$$
v_{F}\left[\vec{\sigma}_{2 D}^{A B} \cdot \vec{p}_{B A}-c^{-1} M_{B A}\right]\left|\psi_{B A}^{*}\right\rangle=E\left|\psi_{B A}^{*}\right\rangle(2.2)
$$

and the same equation with labels (AB, BA) exchanged for another sublattice. Here
$(A B, B A)$ are related to sublattices and refer to the quantities which are obtained by similar transformations with a relativistic exchange matrix $\Sigma_{\text {rel }}^{x}$ e.g., for the momentum operator $\vec{p}$ one gets $\vec{p}_{B A}=\Sigma_{B A} \vec{p} \Sigma_{B A}^{-1} ; \quad v_{F}$ is the Fermi velocity. The vector of $2 \mathrm{D}$ Pauli matrixes comprises of two matrixes $\sigma_{2 D}=\left(\sigma_{x}, \sigma_{y}\right)$. The term $M_{B A}=-\frac{1}{c v_{F}} \Sigma_{B A} \Sigma_{A B}$ is a Majorana-like mass term, where $c$ is the speed of light. It turns out to be zero in the Dirac point $K\left(K^{\prime}\right)$ and gives a very small momentum-dependent correction outside of $K\left(K^{\prime}\right)$. The relativistic exchange operator for tight-binding approximation and accounting of nearest lattice neighbors is given by its action on secondary quantized wave functions on trigonal sublattices $A(B)$ of the system as $[22,24,25]$

$$
\begin{array}{r}
\Sigma_{r e l}^{x}\left(\begin{array}{c}
\hat{\chi}_{-\sigma_{A}}^{\dagger}(\vec{r}) \\
\hat{\chi}_{\sigma_{B}}^{\dagger}(\vec{r})
\end{array}\right)|0,-\sigma\rangle|0, \sigma\rangle(2,3) \\
=\left(\begin{array}{cc}
0 & \Sigma_{A B} \\
\Sigma_{B A} & 0
\end{array}\right)\left(\begin{array}{c}
\hat{\chi}_{-\sigma_{A}}^{\dagger}(\vec{r}) \\
\hat{\chi}_{\sigma_{B}}^{\dagger}(\vec{r})
\end{array}\right)|0,-\sigma\rangle|0, \sigma\rangle,
\end{array}
$$

$$
\begin{aligned}
\Sigma_{A B} \widehat{\chi}_{\sigma_{B}}^{\dagger}(\vec{r})|0, \sigma\rangle & =\sum_{i=1}^{N_{v} N} \int d \vec{r}_{i} \widehat{\chi}_{\sigma_{i} B}^{\dagger}(\vec{r})|0, \sigma\rangle \Delta_{A B}\left\langle 0,-\sigma_{i}\left|\hat{\chi}_{-\sigma_{i}^{A}}^{\dagger}\left(\vec{r}_{i}\right) V\left(\vec{r}_{i}-\vec{r}\right) \widehat{\chi}-\sigma_{B}\left(\vec{r}_{i}\right)\right| 0,-\sigma_{i^{\prime}}\right\rangle, \\
\Sigma_{B A} \hat{\chi}_{-\sigma_{A}}^{\dagger}(\vec{r})|0,-\sigma\rangle & =\sum_{i^{\prime}=1}^{N_{v} N} \int d \vec{r}_{i^{\prime}} \widehat{\chi}_{-\sigma_{i^{\prime}}}^{\dagger}(\vec{r})|0,-\sigma\rangle \Delta_{B A}\left\langle 0, \sigma_{i^{\prime}}\left|\widehat{\chi}_{\sigma_{i^{\prime}}}^{\dagger}\left(\vec{r}_{i^{\prime}}\right) V\left(\vec{r}_{i^{\prime}}-\vec{r}\right) \widehat{\chi}_{\sigma_{A}}\left(\vec{r}_{i^{\prime}}\right)\right| 0, \sigma_{i}\right\rangle .
\end{aligned}
$$

Here interaction $(2 \times 2)$-matrices $\Delta_{A B}$ and $\Delta_{B A}$ are gauge fields (or components of a gauge field). Vector-potentials for these gauge fields are introduced by the phases $\alpha_{0}$ and $\alpha_{ \pm, k}, k=$ $1,2,3$ of $\pi\left(\mathrm{p}_{z}\right)$-electron wave functions $\psi_{\mathrm{p}_{z}}(\vec{r})$ and $\psi_{\mathrm{p}_{z}, \pm \vec{\delta}_{k}}(\vec{r})$ attributed to a given lattice site and its three nearest neighbors (see detail in $[25]), V(\vec{r})$ is the three-dimensional (3D) Coulomb potential, summation is performed on all lattice sites and number of electrons. The introduction of these non-abelian gauge fields was stipulated by a requirement of reality of eigenvalues of the Hamiltonian operator as gauge conditions. In this case, the operator of relativistic exchange gains an additional implicit $\vec{k}$-dependence upon momentum in the case of non-zero values of gauge fields. 


\section{Radiation-resistant model of graphene monolayer}

Carbon nanotube is a cylinder consisting of one rolled-up graphene sheet (SWCNTs) or few concentric rolled-up graphene sheets (MWCNTs). Graphene refers to strongly correlated systems. Band structure of the pure graphene holds a Dirac touching of valent and conduction bands at a Dirac point $K\left(K^{\prime}\right)$ of Brillouin zone. Charge carriers for the pure graphene are electronhole pairs only. Any Dirac fermion described by a complex wave function is a composite fermion. The composition is created by a pair of conventional Majorana and antiMajorana fermions that their wave functions are real ones. Both Dirac and Majorana fermions are particles with spin $1 / 2$. Coulomb screening in the strongly correlated many-body graphene system impedes electromagnatic mixing of Majorana fermions back to Dirac configuration. Statistics of Majorana fermions is non-Abelian one as the rotation on the angle $2 \pi$ leads to change of their wave function phase on $\pi / 4$ [26]. Particles with non-Abelian statistics are called anyons. We describe a production of electron-hole pairs of zero energy in the point $K\left(K^{\prime}\right)$ as an emerging of the Majorana-like and antiMajorana-like fermion excitations $[27,28]$.

Oppositely to the Majorana fermions, eigenfunctions of our Majorana-like model Hamiltonian are complex functions. The number of electron-hole pairs of zero energy and, accordingly the pseudoMajorana quasiparticles are infinite. The Hamiltonian of Majorane-like quasiparticles and anti-quasiparticles in graphene has Majorana mass term only, an ordinary Dirac mass is absent. Features of our graphene model are the chirality of charge carriers due to their Dirac-massless state and dichroism of graphene band structure [28]. Band structure eight-folding at small quasiparticle momenta in this graphene model displays vorticity patterns (see Fig. 2). Electron-hole pairs split up (fissions) into two right- and left-handed triplets of topological defects. Thus, the three-body left (right)-handed Majorana $\mathrm{M}_{p}^{-}$and right (left)- handled antiMajorana $\overline{\mathrm{M}}_{p}^{+}$excitations produce electrons and holes after fusion of three particles in Majorana and antiMajorana configurations respectively. A deconfinement of Majorana quasiparticles happens at sufficiently large values of momenta. Meanwhile, a Fermi velocity tends to zero. Therefore, these Majorana-like excitations are long-lived ones.

(a)

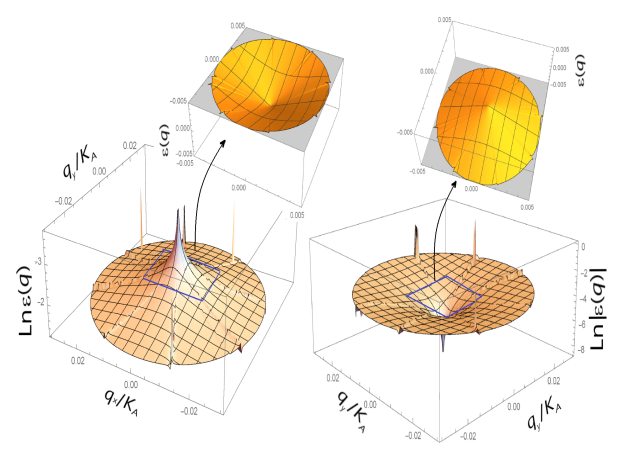

(b)

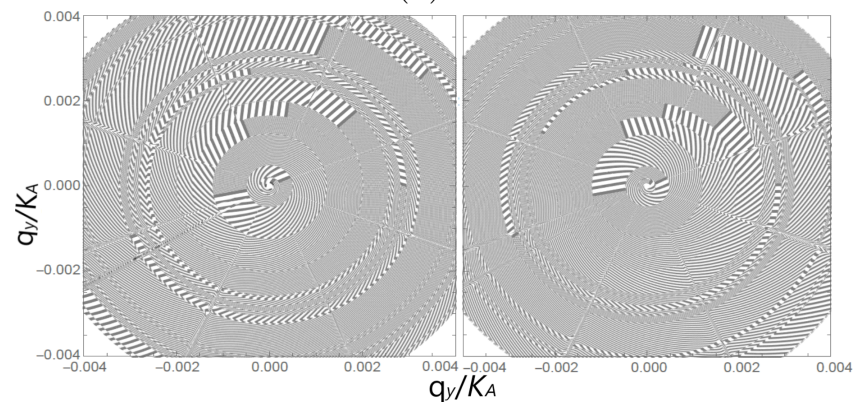

FIG. 2. (color online) (a) Electron (left) and hole (right) valent bands on momentum scale $q / K_{A}=0.02$. (b) A vortex texture in contour plots of electron (left) and hole (right) bands on momentum scales $q / K_{A}=0.002$. The band structure of quasi-relativistic graphene model is calculated taking into account of non-zero gauge field and Majorana-like mass term.

Elastic scattering of electromagnetic radiation quanta occurs with high probability on weakly bound electrons of conduction band [29]. Practically instant recombination of graphene electron-hole pairs in the vicinity the $K\left(K^{\prime}\right)$ point suppresses the Compton effect on electrons and holes in Dirac massless model of graphene.

The Majorana fermion can be presented as a chain of electrons, two of which on the ends of the 
chain are delocalized, while all others electrons are in dimer configurations [30]. Therefore, such topological defects originate Comptom effect of photons scattering on delocalized charge carriers. In spite of gamma-rays the negative charged beta-particles interact with both Majorana quasiparticles and localized electrons. Elastically scattering on topological defects Majorana quasiparticles in the Dirac point, $\gamma$ - and $\beta$-quanta produce flows of deconfined Majorana configurations $\mathrm{M}_{p}^{-}$and $\overline{\mathrm{M}}_{p}^{+}$. Chirality and topological-charge conservation laws prohibit recombination of Majorana particles with charge carriers of other pairs and prevent the charge carriers from escaping outside of graphene plane. The $\overline{\mathrm{M}}_{p}^{+}$state is bound to a neutral knockedout carbon atom $\mathrm{C}^{0}$ and a neutral graphene vacancy $V_{g}^{0}$ appears in a non-filled lattice site. Fig. 3a schematically images the radiation damage of graphene monolayer. The vacancy $V_{g}^{0}$ hosts holes which emerge after fusion Majorana quasiparticles. The charged $V_{g}^{+}$attracts the light $\mathrm{M}_{p}^{-}$. Now, despite the fact that the heavy $\overline{\mathrm{M}}_{p}^{+}$ with $\mathrm{C}^{0}$ moves slowly, $\mathrm{M}_{p}^{-}$await $\overline{\mathrm{M}}_{p}^{+}$in the nonoccupied lattice site and after their fusion the knocked-out atom recovers its lattice location with subsequent repairing of graphene structure (see Fig. 3b). It testifies radiation resistivity of graphene.

\section{Formation of radiation-induced p-n graphene junction: Model}

The mechanism of $\mathrm{p}-\mathrm{n}$ junction formation is schematically presented in Fig. 3c,d. Laserradiation scattering in graphene plane or scattering of photons which the knocked-out carbon atom should emit to transit in nonoccupied graphene lattice site is accompanied by transmission of energy, large enough to deconfine Majorana configurations. The electromagnetic high-frequency radiation, penetrating into higher support layers, create a surface charge wave in support (skin effect). Moreover, the laser pulse or the photons emitted knocked-out atom excite a charge far transfer from neutral support vacancies to positively charged areas of surface wave that a p-doped graphene patch emerges (see Fig. 3c). Pairing the charged vacancy support state $V_{\uparrow(\downarrow)}^{q+}$ and holes $h_{\downarrow(\uparrow)}^{+}$holding opposite-directed spin "up" ("down") and "down" ("up") respectively are produced n-doped graphene patches (see Fig.3d). Then the heavy Majorana state $\overline{\mathrm{M}}_{p}^{+}$is confined in the p-doped graphene, the light state $\mathrm{M}_{p}^{-}$remains in the $\mathrm{n}$-doped graphene patch. As a result, n-p-n graphene junctions are formed. Escaping from plane into graphene-monolayer surface the deconfined Majorana fermions are scattered on impurities if the last exist. Transfering to low-lying orbitals of impurity, the Majorana particles quench a fluorescence of lowlying energy impurity levels because a Pauli principle forbids occupation of the same level by two identical fermions simultaneously. But, the Majorana excitations do not be able to quench a charge far transfer because their disappearing from graphene monolayer is forbidden by the law of topological-charge conservation. Therefore, the p-n graphene junctions are stable.

Further, experimental data which can be explained based on the proposed model of an artificial atom will be presented. The experimental facts will be interpreted based on the proposed model of radiation effects in graphene composite also.

\section{Raman-spectral analysis}

Carbon nanotubes do not fluoresce. In virtue of zero energy of an electron-hole pair, if charge carriers do nor redistribute into electron and hole conduction bands from the Dirac point, than uncompensated electron/hole density in $K\left(K^{\prime}\right)$ is absent and, accordingly, $K\left(K^{\prime}\right)$ of pure graphene monolayer is electrically neutral one. Because of this, Raman scattering of light on graphene charge carriers is absent for defectless graphene monolayers.

Vibrational spectra of carbon nucleus in the electronic terms of non-compensate charge 
(a)

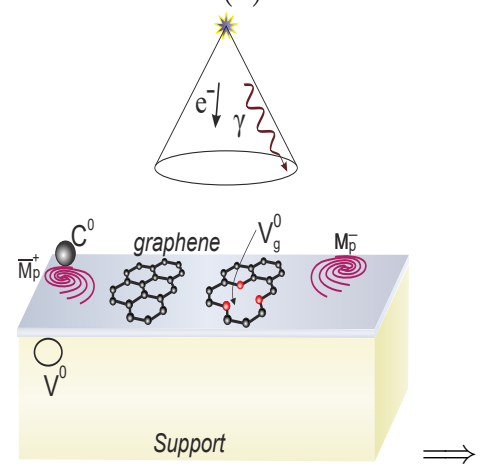

(d)

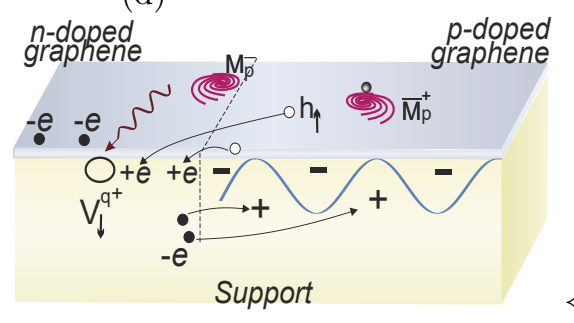

(b)
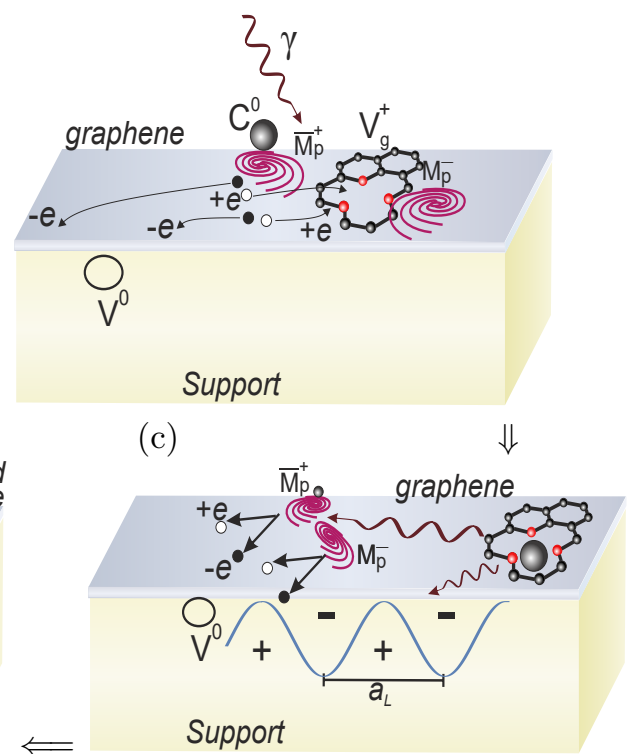

FIG. 3. (color online) A sketch of n-p-n graphene-junction formation induced by radiation. A period of charge support-surface wave is marked by $a_{L}$.

density reveal themselves in the form of the socalled defect $(D)$ and hybridization $(G)$ graphene bands and some other $(2 D, 2 G$ etc.) of Raman spectra. The Raman scattering of light for $\mathrm{p}(\mathrm{n})$ doped graphene monolayer occurs on charge density being in the point $K\left(K^{\prime}\right)$ and the point $\Gamma$ of Brillouin zone. The most intensive spectral bands are the defect $D$ and hybridized $G$ peaks. Meanwhile carbon nuclei vibrate in the terms of gapless and gapped $\pi\left(\mathrm{p}_{z}\right)$-electrons. The existence of the defect $D$ vibration peak in the term of gapless $\pi\left(\mathrm{p}_{z}\right)$-electrons evidences the possibility of electron or hole escape from graphene electronhole pairs to impurity levels if they are presented. The Dirac point losses its electrical neutrality because of remaining free electric charge carriers of opposite sign and the Fermi level of doped graphene monolayer places higher or lower than the Dirac cone point.

The hybridization peak $G$ testifies C nuclei vibrations in the terms of gapped $\pi\left(\mathrm{p}_{z}\right)$-electrons. A value of $\pi\left(\mathrm{p}_{z}\right)$-electron momentum determines a degree of mixing between $\pi\left(\mathrm{p}_{z}\right)$-orbitals and orbitals of hybridized sp-electrons. When adding sp-electrons increase the charge density in the Dirac point and, accordingly, the term of gapped $\pi\left(\mathrm{p}_{z}\right)$-electron is deeper than for gapless $\pi\left(\mathrm{p}_{z}\right)$ electron. It signifies that $\nu_{D}<\nu_{G}$. Table 1 displays features of Raman spectra of MWCNTsamples under investigation. Decorated or nondecorated MWCNT arrays ordered in different ways are deposited on different support.

5.1. Intensity of graphene $D$ and $G$ Raman bands and confinement of charge carriers to graphene plane

Let us study a transfer of MWCNT charge carriers to extrinsic impurity levels. An ensemble of original MWCNTs most intensively scatters a light in the Raman spectral bands $D$ and $G$ with maximums at frequencies 1328 and $1566 \mathrm{~cm}^{-1}$. It signifies that the charge density in the point $K\left(K^{\prime}\right)$ is not compensated. A Van der Waals attractive forces between hydrophilic carboxyl residues of stearic acid and MWCNTs 
Table 1. Raman characteristic frequencies $\nu_{D}, \nu_{G}$ and band-intensity maxima $I_{D}, I_{G}$ for graphene monolayers of origin multiwalled carbon nanotubes and their different bundles deposited on different support at different wavelengths $\lambda$ at RT.

\begin{tabular}{|c|c|c|c|c|c|}
\hline Support & Sample & $\nu_{D}, \mathrm{~cm}^{-1}$ & $\nu_{G}, \mathrm{~cm}^{-1}$ & $I_{D} / I_{G}$ & $\lambda, \mathrm{nm}$ \\
\hline \multirow{8}{*}{ Si hydrophilized } & origin MWCNTs & 1328 & 1566 & 1.165 & \multirow{4}{*}{532} \\
\hline & micellar MWCNTs & $-*$ & 1575 & 0 & \\
\hline & $\begin{array}{l}\text { micellar oligonucleotide-encased } \\
\text { MWCNTs }\end{array}$ & \multirow[t]{2}{*}{1340} & \multirow[t]{2}{*}{1572} & 1.02 & \\
\hline & $\begin{array}{l}\text { oligonucleotide-encased } \\
\text { MWCNT one-monolayer film }\end{array}$ & & & 0.81 & \\
\hline & $\begin{array}{l}\text { three-monolayer non-decorated } \\
\text { MWCNT LB-film }\end{array}$ & $\begin{array}{l}1400- \\
1403\end{array}$ & 1586 & 0.25 & 355 \\
\hline & \multirow{5}{*}{$\begin{array}{l}\text { two-monolayer organometallic- } \\
\text { compound-decorated MWCNT }\end{array}$} & - & - & - & 532 \\
\hline & & & & & \\
\hline & & $\begin{array}{l}1398- \\
1400\end{array}$ & $\begin{array}{l}1597- \\
1600\end{array}$ & 0.1875 & 473 \\
\hline \multirow{3}{*}{ AOA hydrophilized } & & \multirow{2}{*}{ - } & \multirow{2}{*}{ - } & \multirow{2}{*}{ - } & \\
\hline & & & & & 532 \\
\hline & \multirow{2}{*}{$\begin{array}{l}\text { two-monolayer non-decorated } \\
\text { MWCNT LB-film }\end{array}$} & \multirow[t]{2}{*}{1353} & \multirow[t]{2}{*}{1573} & 0.75 & \multirow[t]{2}{*}{473} \\
\hline AOA hydrophobized & & & & 0.167 & \\
\hline
\end{tabular}

* - Hyphen means that a corresponding peak is absent.

form inverse micelles of stearic acids, hydrophobic alkyl hydrocarbon chain of which are directed outside. Meanwhile, MWCNTs are placed inside the micelles. Micellar MWCNTs were deposited on hydrophobized or hydrophilized surface of AOA and hydrophilized Si. Since the peak $D$ disappears practically for samples of micellar MWCNTs, electric charge carriers are absent in the Dirac point and the Fermi level is in the Dirac band touching. It signifies that micellar MWCNTs inside inverse micelles are self-organized into a high-ordered ensemble with practically defectless structure. According to Table 1, the peak $D$ appears again in the spectra of LB-films formed from inverse micelles with nondecorated MWCNTs inside after their deposition on the support. Repulsion between hydrophobic groups of LB-film and hydrophilic groups of the support leads to emerging of graphene-lattice deformation defects. Localization of charge carriers from MWCNTs electron-hole pairs on such impurity levels originates non-compensated charge density in $K\left(K^{\prime}\right)$ and $\Gamma$. Meanwhile, the Fermi level shifts from the touching point of graphene electron and hole conduction bands. Owing to the law of spirality conservation, the free charge carriers do not annihilate with other remaining charge carriers of opposite sign. Because the number of Majorana particles is infinite large, the electric charge transfer happens to impurity levels of all defects. The charge carriers in graphene materials do not recombine with impurity and support-defect states owing to the law of topological-charge conservation. As Table 1 demonstrates, the height of peak $D$ increases at rise of the number of impurity levels, electron (hole) density of which forms bound states (excitons) with graphene charge carriers. A quantity of states bound to impurity levels is determined by electrocapillary effects (electrocapillary forces). The hydrophobic LBMWCNT-film extends on hydrophobic surface under the action of electrocapillary forces uniformly. Meanwhile CNTs straighten up moving 
away the support. On the hydrophilic surface the action of electrocapillary forces bending the hyrdophobic film leads to emerging of regions where CNTs abut on the support very closely. Because of this the intensity ratio $I_{D} / I_{G}$ for the peaks $D$ and $G$ of scattering in the hydrophobic LB-film with two non-decoratedMWCNT monolayers is significant less in the case of hydrophobic AOA support $\left(I_{D} / I_{G}=\right.$ $0.167)$, than in the case of hydrophilic AOA support $\left(I_{D} / I_{G}=0.167\right.$, see Table 1$)$. Similarly, micellar MWCNTs which do not touch the support surface, have not the defect levels and, accordingly, the peak $D$ is absent in their spectra. Oppositely, due to a strong contact between individual bend original MWCNTs and Si support the ratio takes a high value: $I_{D} / I_{G}=1.165$.

So, the graphene charge carriers are held in the vicinity of the MWCNT-surface. This confinement phenomenon occurs due to fact that electrons and holes are produced in Majorana and antiMajorana configurations. When flatting the energy band prevents the fusion of Majorana and antiMajorana quasiparticle due to zero Fermi velocity: $v_{F} \rightarrow 0$ at high momenta. At that, the infinitely large Majorana force confines the graphene charge carriers inducing transitions of the charge carriers from high-lying levels to lowlying ones [33]. The latter precludes pairing between the graphene charge carriers and the opposite-charged impurity electrons (holes) on the high-lying impurity levels.

The frequencies $\nu_{D}$ and $\nu_{G}$ of characteristic vibrations do not depend on the height of $D$ - and $G$-peaks and, accordingly, do not depend on the number of graphene charge carriers localized on the impurity levels of a given type. For example, according to Table $1, \nu_{D}=1353 \mathrm{~nm}$ and $\nu_{G}=1573 \mathrm{~nm}$ at laser excitation $473 \mathrm{~nm}$ for MWCNTs which are arranged on both hydrophilized and hydrophobized AOA surfaces. Another example, $\nu_{D}=1340 \mathrm{~nm}$ and $\nu_{G}=1572 \mathrm{~nm}$ at laser excitation $532 \mathrm{~nm}$ for micellar oligonucleotideencased MWCNTs and the LB-monolayer consisting of oligonucleotide-encased MWCNTs (see Table 1). $D$ - and $G$-bands position for
MWCNTs at given laser wavelength and at the same type of the impurity level indicates that the graphene lattice-deformation defects linked to the Dirac point and, accordingly, do not take external charge carriers on their energy levels.

\subsection{Quenching effect}

The oligonucleotide cover of MWCNTs does not fluoresce because the Majorana quasiparticles occupy oligonucleotide low-lying levels. Thus, the scattering of electromagnetic radiation in aromatic compounds deposited on graphene surface is quenched. The charge carriers of graphene material do not quench high-lying levels of support defects because the Majorana particles can be just in graphene plane. One observes sharp decrease of $I_{D} / I_{G}$ at $\lambda_{e x}=355 \mathrm{~nm}$ in comparison with the excitation $\lambda_{e x}=473,532 \mathrm{~nm}$ for the defective support and samples (see Table 1). It testifies also that the pseudo-Majorana states transfer from the impurity back to the graphene monolayer because they are not able to hybridize with high-lying impurity orbitals.

\subsection{Emerging graphene $D, G$ bands at support-defect charging}

An electromagnetic field penetrates into a sample to a definite depth (skin effect). The penetration depth of a electromagnetic field is about a half of the wavelength. Penetrating into a sample, the electromagnetic quanta excite surface states. A superstructure of graphene monolayer is induced due to the skin effect. Let us prove that electrical fields of charged support vacancies destroy the graphene supercells, and remaining supercells represent artificial atoms. To make it we study Raman spectra of the high-ordered LBmonolayers which consist of MWCNTs decorated by the organometallic complexes and are arranged on $\mathrm{Si}$ or nanoporous anodic alumina.

The value $\nu_{D}$ depends on laser wavelength values $\lambda_{e x}$ so that $\nu_{D}$ increases with decrease of $\lambda_{e x}$ as Table 1 demonstrates. At the average it grows also with the decrease of $\lambda_{e x}$ (see 
Table 1). The Raman frequencies are higher if the band structure of MWCNT-samples is distorted less. For example, at the same laser wavelength $\lambda_{e x}=532 \mathrm{~nm} \nu_{G}=1566$ and $1575 \mathrm{~cm}^{-1}$ for chaotic arranged origin and micellar MWCNTs respectively. The highest charge density in the Dirac point is achieved in the LB-films of decorated MWCNTs as $\nu_{G}=1587-1600 \mathrm{~nm}$ at $\lambda_{e x}=473 \mathrm{~nm}$ and $\nu_{G}=1586 \mathrm{~cm}^{-1}$ at $\lambda_{e x}=$ $355 \mathrm{~nm}$ for the decorated MWCNTs and the LBfilm of non-decorated MWCNTs respectively on Si support.

Thus, our model predicts and the experimental data testify that variation of p-doping conditions stems from the variation of superlattice potential. The frequencies $\nu_{D}$ and $\nu_{G}$ depend on $\lambda_{e x}$ because the parameters of surface wave determine the graphene supercells.

\subsection{CNT-enhanced scattering light in metal-containing DTP LB-films}

The peaks $D$ and $G$ are not observed for decorated MWCNTs suspended на AOA nanopores at $\lambda_{e x}=473,532 \mathrm{~nm}$ and for MWCNTs deposited on Si at $\lambda_{e x}=532 \mathrm{~nm}$. The absence of characteristic bands of MWCNTs in Raman spectra testifies the practically perfect crystal structure of decorated-MWCNTmonolayers with neutral Dirac point (see Table 1). The charge carriers do not be transferred along the LB-DTP-film at laser excitation with wavelength $473 \mathrm{~nm}$ but accumulate in sites of contact between molecules Fe(II)DTP and Si surface because of disruption of double-bound chain with the subsequent localization of charge carriers. The value of $I_{D} / I_{G}$ equals to 0.1875 at $\lambda_{e x}=473 \mathrm{~nm}$ and is comparable with $I_{D} / I_{G}=$ 0.25 at $\lambda_{e x}=355 \mathrm{~nm}$ for decorated and nondecorated MWCNTs respectively that indicates the long charge transport to high-lying levels where graphene charge carriers are not able to exist. The graphene charge carriers holding in the electric field of charged high-energy surface states form the artificial atoms. Since a MWCNTplasmon enhancement of light scattering for the
DTP LB-film on $\mathrm{Si}$ at $473 \mathrm{~nm}$ disappears, practically all free charge carriers enter the artificial atoms. On the contrary, the Raman spectra for the decorated MWCNTs suspended on AOA nanopores do not distinguished at laser excitations with wavelengths 473 and $532 \mathrm{~nm}$ due to the absence of support and, accordingly, of charged support defects.

Different-type MWCNT arrays shield electric fields with different efficiency. The quantity of charged support vacancies is much less at more effective screening. Therefore, the artificial atoms and the potential electrically confining these p-doped graphene patches are much bigger at the effective screening of support. Nuclei $\mathrm{C}$ oscillate much faster for the deep confining potential than shallow one. For example, accordingly to Table 1 , the values of $\nu_{D}$ and $\nu_{G}$ are larger for the MWCNT array decorated by organometallic-compound than oligonucleotide-encased one because the former shields better than the later.

\section{Impedance analysis}

Dielectric spectra of non-exposed structures under investigation are presented in Fig. 4. Unlike to the graphene-like materials the dielectric spectra of AOA do not have specific dielectric Cole-Cole plots with a diffuse element or Warburg impedance $W$. Since the Warburg impedance is absent for AOA and, consequently, aluminium-electrodes oxidation does not happen, the fabricated nanoheterostructures are stable. The Cole-Cole plot for metal-containing dithionylpyrrole LB-films testifies both the Warburg impedance element $W$ of diffuse layer in which the electrochemical reactions with mass transfer proceed and an electric capacity of Helmholtz double layer. For the dithionylpyrrole LB-films, this oxidation-reduction potential stipulated by change of molecular-group charge state arises due to self-redox activity of pyrrole residue and transport of electrical charge along the chain of conjugated double bonds. MWCNTs, as LB-DTP-films, are redox active themself 
according to the Cole-Cole plot of spectrum 3 with $W$ in Fig. 4. The change of charge state of $\mathrm{K}\left(\mathrm{K}^{\prime}\right)$ point is associated with mass transfer that Kitaev-like chains with Majorana end states emerge. Meanwhile, plasma oscillations of produced electron-hole pairs shield electric field of charged electrodes.

According to Bode capacity plots 1, 2, and 4 in Fig. 5a for irradiated and nonirradiated organometallic-compound LB-films a dipole relaxation of radiation-vacancies clusters is observed simultaneously with the increase of shielding degree in two weeks after irradiation by IRS ${ }^{137} \mathrm{Ce}$. The sharp increase of shielding degree indicates that the number of free charge carriers increase extremely. Because electron-hole pairs are charge carriers in graphene and graphenelike materials, the increase of free charge carriers density indicates localization of holes or electrons from electron-hole pairs after irradiation. The incoming particles can collide with electrons only. Since the atoms C are knocked out, holes (vacancies) localized in the Dirac point remain.

The charge density of vacancies ensemble has a form of atom-like density of localized holes surrounded by delocalized electron density. The dipole relaxation of these individual radiationvacancy ensembles disappears for long enough time about three months according to comparison of dielectric spectra 2 and 3 in Fig. 5a. Emerging only two weeks latter after irradiation the individual atom-like vacancy arrays indicate a decay of very large cluster of radiation vacancies into the small atom-like arrays (artificial atoms) in graphene-like monolayer plane during selfrepairing processes.

Because the high energy of gamma- and beta-rays produced by IRS the atom-like ensembles of radiation vacancies are produced in excited state with non-occupied both valence and intrinsic orbitals by the mechanism proposed in Section 4. Overlapping the external orbitals of such artificial atoms in excited state produce large radiation-clusters of vacancies. Hydrate complexes of atom-like structures formed by vacancies are produced after transition of the last into the ground state after two weeks according

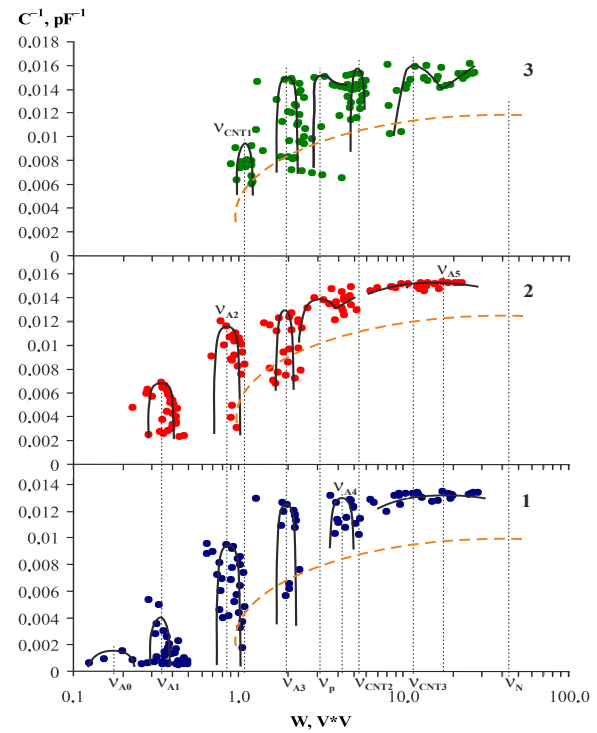

FIG. 4. (color online) Dielectric spectra 1 for nanoporous AOA, 2 and 3 for наногетерострукtyp based on Ce-containing nanocyclic compound $\mathrm{Fe}(\mathrm{II}) \mathrm{DTP}$ and on decorated MWCNTs, respectively. Resonance frequencies $\nu$ of Cole-Cole plots in the spectra are marked as $\nu_{A i}, i=0, \ldots, 5$ for AOA; $\nu_{P}$ for the organometallic compound and $\nu_{C N T i}, i=1,2,3$ for MWCNTs. A Nyquist plot of impedance for the measuring circuit is marked by a yellow dashed line with $\nu_{N}$

the comparison of the Bode plots 1 and 2, Fig. 5a. This is evidence that the external orbitals in the ground state is not overlapped. The decay of large radiation cluster into the separated artificial atoms (electrically confined quantum dots) is testified by the corresponding resonance at the frequency $\sim 280 \mathrm{kHz}$ for the metal-containing LBfilm. A lifetime of individual quantum dot is very large so that the dots exist for one-two months.

Mobile interstitial carbon atoms being near monolayer plane can form chemical cross-links with hydrocarbon alkyl chains. According to comparison of the frequency dependencies 3 and 4 in Fig. 5a, after decay of artificial atoms the variation of electrophysical properties of metalcontaining dithionylpyrrole LB-films is reduced practically to a weak increase of original value of the sensor electric capacity. The weak increase is 


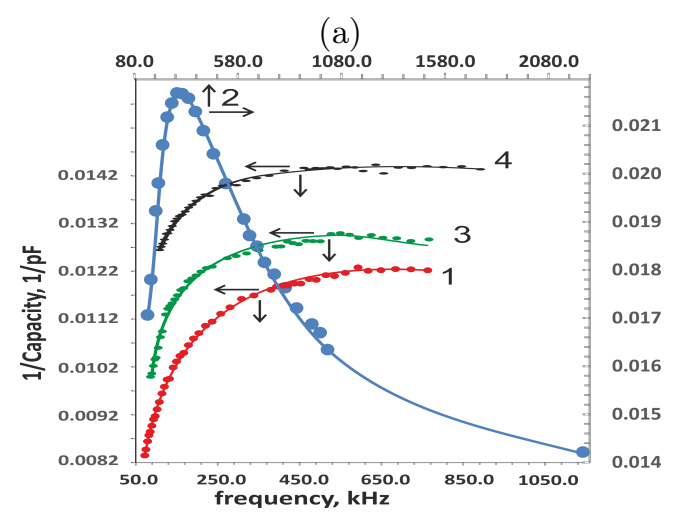

(b)

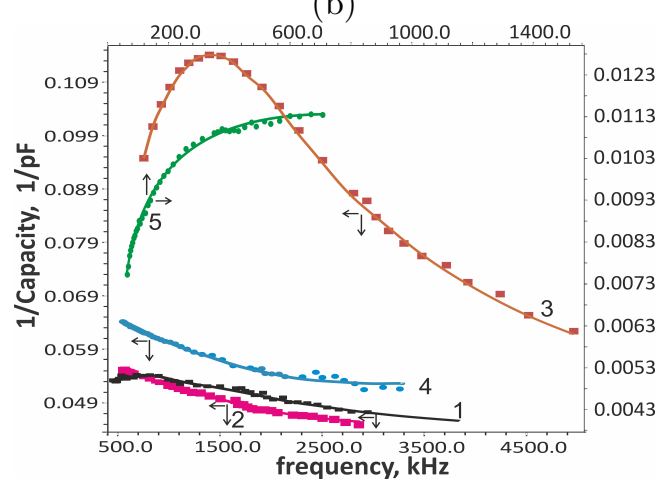

FIG. 5. (color online) Frequency dependences of electrical capacity: (a) of Fe-containing dithionylpyrrole LB-film and (b) MWCNTs decorated by nanocyclic complexes of $\mathrm{Fe}$ and $\mathrm{Ce}$ with dithionylpyrrole ligands. (a) 1 - immediately after irradiation, 2 - in two weeks after irradiation, 3 - in three months after irradiation, 4 - before irradiation; the duration of irradiation was 1 hour. (b) 1 - immediately after irradiation, 2 - in 24 hours after irradiation, 3 - in three weeks after irradiation, 4 - in four months after irradiation, 5 - before irradiation; the duration of irradiation was 3 hours.

stipulated by a small destroying of the doublebound chains by the cross-links with near-lying alkyl hydrocarbon chains and, respectively, by an attenuation of shielding effect .

A mechanism of MWCNT radiation resistivity is similar to the mechanism for metal-containing dithionylpyrrole LB-film. The dielectric spectra of irradiated LB-films of MWCNTs decorated by nanocyclic complexes of $\mathrm{Ce}$ and octahedral high-spin $\mathrm{Fe}$, are presented in Fig. 5b. According to the Bode plot 3,
Fig. 5b, the dipole relaxation of hydrated atomlike clusters of radiation vacancies occurs at higher frequencies (about $1320 \mathrm{kHz}$ ) than in the dithionylpyrrole films. This is due to the restriction of the artificial atom size by CNT diameter value. According to the Bode plots $1-4$, Fig. 5b, the decay of excited radiationinduced artificial-atom aggregates occurs in three weeks after irradiation, while the restoration of electrophysical properties of MWCNTs occurs in three month after the irradiation when quasistationary artificial atoms disappear. The shift of the maximum in the frequency dependence 1, Fig. 5b towards low frequencies is registered immediately after irradiation also. This shift indicates the formation of atom-like clusters of the vacancies under the action of $\gamma$ - and $\beta$-rays in the metal-containing dithionylpyrrole LB-monolayers decorating MWCNTs.

Decrease of electric capacity of nonirradiated MWCNTs from $85.2 \mathrm{pF}$ to $18.5 \mathrm{pF}$ is registered immediately after three-hour irradiation. Accordingly, the increase of the intensity in the Bode plot 1 in respect of the plot 5 in Fig. 5b testifies the high shielding efficiency of MWCNTs-network and, accordingly, its high electric conductivity. It signifies that in the addition to the radiation-resistivity mechanism for metal-containing dithionylpyrrole LB-film there is cross-connections between graphene monolayers of MWCNT and interstital knockedout atoms $\mathrm{C}$ through chemical bounds into a high-shielding network.

Shielding effects of the LB-films is revealed also as an intensity change of Compton scattering and as an increase of intensity of secondary electrons spectra in the detector of $\gamma$-rays.

Thus, as the quasi-relativistic graphene model predicts, the experimental study fulfilled discovers that there exists a Compton effect at irradiation of the graphene-like material. Further, we propose a model of the Compton effect enhanced by a collapse of artificial heavy atomic states in graphene monolayers. 


\section{Enhancement of Compton effect in graphene}

Accordingly to the experimental data, a time of dipole relaxation is about $0.7 \mu \mathrm{s}$. Such large relaxation times testify that the $\mathrm{p}-\mathrm{n}$ junctions are sufficiently big. The relaxation process is observed only after a time $\tau_{C}$ about $2-3$ weeks after irradiation. Therefore, $\tau_{C}$ is time for which the cluster of original artificial atoms decays. It signifies that the original artificial atoms are chemically linked in the cluster with a life time $\tau_{C}$. A life time $\tau_{J}$ of free artificial atoms appearing after decay of the large radiation cluster is much longer, more than a month. After this period the electrochemical properties of the samples are recovered. Since the orbitals of original artificial atoms overlap, they are much larger than for the free $\mathrm{p}-\mathrm{n}$ junctions. But, large atoms with ultra-heavy nuclei are in a supercritical regime that is the electronic orbitals, starting with $1 \mathrm{~S}$ state, sink into the Dirac continuum until an atom number $Z$ is reduced to a critical value. This process is known as atomic collapse [34]. Opposite to the supercriticality of an atom, the orbitals of artificial atom sink into the Majorana continuum until $Z$ is reduced to a critical value. An enormous number of secondary delocalized electrons emerging after fusion between sunken $M_{p}^{-}$-vortices and $\bar{M}_{p}^{+}$ anti-vortices provides sharp increase of the photon elastic scattering cross-section. The very large electron density in the irradiated samples is registered by scintillator as significant bend of direction of propagation of $\gamma$-quanta from the original once. After the atom-state collapses the hybridized charge carriers are on high-lying discrete energy levels of $\mathrm{p}-\mathrm{n}$ graphene junction. Such cluster is unstable due to its high energy accordingly to the electrochemical study.

\section{Discussion and conclusion}

Let us estimate the performance of graphene composites under investigation as radiation detectors. Since very much low-energy electron- hole pairs are produced in graphene monolayer after the scattering of electromagnetic quanta on the topological defects, the scattering crosssection $S_{M}^{f}$ on the topological defects is much smaller than a scattering cross-section $S_{e-h}^{f}$ of free electron-hole pairs:

$$
k_{M^{f}}=S_{e-h}^{f} / S_{M}^{f} \gg 1 .
$$

Let us call the value $k_{M^{f}}$ as an enhancement coefficient due to production of free electron-hole pairs. Sub-picosecond recombination of electrons and holes attenuates this enhancement so that $k_{M^{f}}>1$. However, the cross-section of the elastic scattering of photons on the secondary delocalized electrons from the artificial collapsed atom is a significant effect. Let us suppose that the cross-section of Comptom scattering on the secondary electrons is larger than the crosssection of the scattering on the electrons from electron-hole pairs by $R_{A}^{2} / R_{e-h}^{2}$. Here $R_{e-h}$ is a radius of free electron-hole pair of about an atom size, $R_{A}$ is a radius of nanosize artificial atom. Besides, photons emitted by knocked $\mathrm{C}^{0}$ during its occupation of free lattice site form the atomlike structures in the nearest monolayers also. Because of that, the final enhancement coefficient $k_{\gamma}$ of Compton scattering is also proportional to a number of graphene monolayers $N_{w}$ :

$$
k_{\gamma}=k_{M^{f}} N_{w} R_{A}^{2} / R_{e-h}^{2} \gg 1 .
$$

Substituting (8.1) into (8.2), one get

$$
k_{\gamma}=N_{w} S_{A} / S_{M}^{f} \gg 1
$$

as $S_{e-h}^{f} \sim R_{e-h}^{2}$. Here $S_{A}$ is a scattering crosssection of artificial atom. In the case of LBfilms contained from MWCNTs, it is necessary to take into account the MWCNT-layer number. This shielding of radiation defect by avalanchelike increase of the number of free charge carriers comes to minimum the radiation destruction of graphene-like material.

Beta-particle detection. Opposite to gammarays detection, Coulomb interaction of negativecharged beta-particle occurs not only with free Majorana particles, but also with bound electronhole pairs. Therefore, the enhancement coefficient 
of scattering by graphene plane due to collisions with Majorana quasiparticles is

$$
k_{M}=k_{M^{f}}+k_{M^{b}}=k_{M^{f}}+S_{e-h}^{b} / S_{M}^{b} \gg 1
$$

where $S_{M}^{b}$ and $S_{e-h}^{b}$ are the cross-sections for the scattering on the bound Majorana states and on the bound electron-hole pairs, respectively. Although $S_{e-h}^{b} / S_{M}^{b} \gg 1$, because of the subpicosecond electron-hole recombination the ratio $k_{M}$ is not much higher than $k_{M}^{f}$. Further enhancement of $\beta$-scattering occurs by avalanchelike increase of charge carriers number by the mechanism similarly as for $\gamma$-rays. Besides, $\beta^{-}$particles can additionally interact with enormous number electrons localized in n-doped graphene patch. Therefore, assuming that $S_{e-h}^{b} \approx S_{e-h}^{f}$, the enhancement coefficient $k_{\beta}$ of Coulomb scattering of $\beta$-particles on $N_{w}$ graphene planes is determined by the following expression:

$$
\begin{array}{r}
k_{\beta}=k_{M} N_{w} R_{A}^{2} / R_{e-h}^{2}+k_{n d} \\
=N_{w} S_{A}\left(\frac{1}{S_{M}^{f}}+\frac{1}{S_{M}^{b}}\right)+k_{n d} \gg 1
\end{array}
$$

where $k_{n d}$ is the enhancement coefficient of the elastic $\beta$-scattering due to the existence of $n$ doped graphene patch.

So, the defects created under the action of $650 \mathrm{keV}$ gamma- and $1 \mathrm{MeV}$ beta-radiation do not lead to radiation destruction both twomonolayer LB-films of MWCNTs decorated by nanocyclic organometallic complexes and the ultra-thin metal-containing conductive oligomer LB-films. Collisions of bombarding particles with delocalized electron density of the topological defects in graphene material form the flows of electron-hole pairs. Radiation resistance of synthesized graphene-like materials is provided by stabilization of the band structure due to confinement of the hole part of electron-hole pairs density in of $K\left(K^{\prime}\right)$-point charged due to electron/holes fluxes from the topological defects. At that, the radiation clusters consisting of nanosize artificial atoms (n-p-n junctions) are created in the graphene monolayer. The sink of charge carriers into the Majorana continuum with a collapse of atomic states occurs. The crosssection of elastic scattering of $\gamma$-quanta grows effectively due to existence of these secondary electrons. The overlap of high-lying orbitals of p-n junctions produces quasi-stable "crystal" (cluster) of the artificial atoms. Dipole relaxation for the artificial crystal provided by the displacement of its artificial atoms happens at high frequencies. When the crystal decays, the reorientation of dipole moments of the individual artificial atoms takes place at low frequencies.

\section{References}

[1] Y.-f. Xiong et al. Ultrahigh Responsivity Photodetectors of Two-dimensional Covalent Organic Frameworks Integrated on Graphene. Advanced Materials. 32, 1907242 (2020).

[2] V.L. Gurachevski. Radiation control : Physical foundation and instrumental equipment. (Radiology Institute Press, Minsk, 2014). (in Russian)

[3] J. Lindhard. Influence of crystal lattice on motion of energetic charged particles. Matematisk-Fysiske Meddelelser Det Kongelige Danske Videnskabernes Selskab. 34, 1-65 (1965).

[4] H. Huang et al. Graphene damage effects on radiation-resistance and configuration of coppergraphene nanocomposite under irradiation: A molecular dynamics study. Scientific Reports. 6, 39391 (2016).

[5] Y. Kim et al. Radiation resistant vanadiumgraphene nanolayered composite. Sci. Rep. 6, 24785 (2016).

[6] L.S. Novikov, E.N. Voronina. Prospect of nanomaterials applying in cosmic technics. (University book, Moscow, 2008). (in Russian)

[7] G. Chai, H. Heinrich, L. Chow, T. Schenkel. Appl. Phys. Lett. 91, 103101 (2007).

[8] M.C. Evora et al. Effect of electron beam and 
gamma rays on carbon nanotube yarn structure. Materials Research. 20(Suppl. 2), 386(2017).

[9] S.N. Shul'ga et al. On classical and quantum effects at scattering of ultrarelativistic electrons in ultrathin crystal. Nucl. Instrum. Methods Phys. Res. Sect. B. 402, 16(2017).

[10] Y. Takabayashi, Y.L. Pivovarov, T.A. Tukhfatullin. First observation of scattering of sub-GeV electrons in ultrathin Si crystal at planar alignment and its relevance to crystalassisted 1D rainbow scattering. Phys. Lett. B. 785, 347 (2018).

[11] M. Ćosić, S. Petrovi, N. Nešković. Quantum rainbows in positron transmission through Carbon Nanotubes. Atoms. Vol. 7, 16 (2019).

[12] B. Li et al. The effect of gamma ray irradiation on the structure of graphite and multiwalled carbon nanotubes. Carbon. 60, 186 (2013).

[13] J. Mao et al. Realization of a tunable artificial atom at a supercritical charged vacancy in graphene. Nature Phys. 12, 545 (2016).

[14] A.V. Korol, A.V. Solov'yov, W. Greiner. Channeling and Radiation in Periodically Bent Crystals. (Springer-Verlag, Berlin, Heidelberg, 2014).

[15] V.V. Klimov, V.S. Letokhov. Monochromatic $\gamma$-radiation emitted by a relativistic electron moving in a carbon nanotube. Phys. Lett. A. 226, 244 (1997).

[16] X. Artru et al. Carbon nanotubes and fullerites in high-energy and X-ray physics. Phys. Rep. 412, 89 (2005).

[17] C.-H. Liu et al. Graphene photodetectors with ultra-broadband and high responsivity at room temperature. Nat Nanotechnol. 9(4), 273 (2014).

[18] A. Agrawal, G.H. McKinley. Nanobubble formation at the solid-liquid interface studied by atomic force microscopy. Materials Research Society Symposium Proceedings. 899E, Paper 0899-N07-37 (2006).

[19] Nucl. Data Sheets. 108, 2173 (2007).

[20] T.E. Konstantinova, E.I. Lyafer. Hydrostatic compaction pressure influence on sintered spodumene pyroceramics properties. In: High pressure science and technology. Ed. W.T. Trzeciakowski. (World Scientific Publishing, Singapore, 1996). Pp. 118-120.

[21] I.I. Abramov et al. A method of impedance calculation for impedimetric sensor with interdigital structure Petersburg electronics J.
4(73), 59-67 (2012). (in Russian)

[22] H.V. Grushevskaya, G.G. Krylov, In: Graphene Science Handbook: Electrical and Optical Properties. Vol. 3. Chapter 9. Eds. M. Aliofkhazraei et al.Taylor and Francis Group, CRC Press, USA, UK, 2016.

[23] H. Grushevskaya, G. Krylov. Vortex Dynamics of Charge Carriers in the Quasi-Relativistic Graphene Model: High-Energy k > p > Approximation Symmetry 12, 261 (2020).

[24] H.V. Grushevskaya, G. Krylov. Semimetals with Fermi Velocity Affected by Exchange Interactions: Two Dimensional Majorana Charge Carriers. J. Nonlin. Phenom. in Complex Sys. 18, 266 (2015).

[25] H.V. Grushevskaya et al. Symmetry of Model $\mathrm{N}=3$ for Graphene with Charged PseudoExcitons. Int. J. Nonlin. Phenom. in Complex Sys. 18, 81 (2015).

[26] C.W.J. Beenakker. Search for Majorana Fermions in Superconductors. Annu. Rev. Condens. Matt. Phys. 4, 113-136 (2013).

[27] H.V. Grushevskaya, G.G. Krylov. Massless Majorana-like charged carriers in twodimensional semimetals. Symmetry. 8, 60 (2016).

[28] H.V. Grushevskaya, G.G. Krylov. Increasing Spin-Orbital Coupling at Relativistic Exchange Interaction of Electron-Hole Pairs in Graphene. Semiconductors. 52, 1879 (2018).

[29] E.A. Murzina. Interaction of radiation with matter. (KDU, Moscow, 2007). (in Russian)

[30] A.Y. Kitaev. Unpaired Majorana fermions in quantum wires. Physics Uspekhi. 44 (Suppl.), 131 (2001).

[31] Ch. Gutierrez et al. Klein tunnelling and electron trapping in nanometre-scale graphene quantum dots. Nature Physics. 12, 1069 (2016).

[32] V.A. Starodubtsev, T.V. Fursa, N.N. Zausaeva. Charge electrification of irradiated dielectrics and its effect on incident flux. J. Electrostatics. 20, 341 (1988).

[33] H.V. Grushevskaya, G. Krylov. Two-Dimensional braiding of two-dimensional majorana fermions : manifestation in band structure of graphene. Int. J. Nonlinear Phenom. Compl. Systems. 22, 41 (2019).

[34] Y.B. Zel'dovich, V.S. Popov. Electronic structure of superheavy atoms // Sov. Phys. Usp. Vol. 14, 673-694 (1972). 